\title{
Ärzte-eigene Datensammlung: Rolle und Verantwortung der Kantone
}

\author{
Solide gesundheitsökonomische Daten bilden die Basis für eine einheitliche Tarif- \\ struktur im Schweizerischen Gesundheitswesen. Am 29.3.2012 hat NewIndex im \\ Rahmen der Tagung «Ärzte-eigene Datensammlung: Rolle und Verantwortung der \\ Kantone» das Projekt OBELISC den Verantwortlichen der Kantonalen Ärztegesell- \\ schaften und der TrustCenter präsentiert.
}

Renato Laffranchi, Philip Moline

NewIndex AG

Korrespondenz:

Dr. Renato Laffranchi

NewIndex AG

Leberngasse 19

CH-4600 Olten

renato.laffranchi[at]newindex.ch
NewIndex AG sammelt seit 2004 Abrechnungsdaten der freipraktizierenden Ärzteschaft in der Schweiz. Diese Daten, die jeder einzelne Arzt über das TrustCenter zur Verfügung stellt, werden aggregiert (verdichtet) und dienen als Grundlage für den Praxisspiegel. Jeder einzelne Arzt hat somit die Möglichkeit, sich in einem Benchmark mit Kolleginnen und Kollegen der gleichen Fachrichtung zu vergleichen und sich zudem vor ungerechtfertigten Rückforderungen der Versicherer besser zu schützen. Gesamtschweizerisch verarbeiten 10 TrustCenter ärzteeigene Daten und schaffen so einen Datenpool, der als Instrument für Tarifverhandlungen mit den Versicherern dient und im Falle von Wirtschaftlichkeitsverfahren zur Verfügung steht, betonte Andreas Häfeli, Verwaltungsratspräsident der NewIndex.

Urs Stoffel, Präsident der Ärztegesellschaft Zürich, unterstrich die Wichtigkeit der anonymisierten, konsolidierten und aggregierten Daten, welche die NewIndex auswertet und analysiert. «Diese Daten sind unerlässlich für die Plausibilisierung und Steuerung der Leistungs- und Kostenvereinbarung

\footnotetext{
Newlndex AG

Die Firma Newlndex wurde im Jahr 2000 im Hinblick auf die künftigen tarifarischen Anforderungen unter TARMED gegründet und ist zu $100 \%$ im Besitz der Kantonalen Ärztegesellschaften, der FMH und der Ärztekasse. Zweck von NewIndex ist die Schaffung eines gesamtschweizerischen Datenpools, um ärzteeigene Daten für die Tarifverhandlungen mit den Versicherern zu generieren und um Ärztinnen und Ärzten ein Instrument für allfällige Wirtschaftlichkeitsverfahren zur Verfügung zu stellen.

$\mathrm{Zu}$ den erfolgreichen Projekten der Newlndex AG gehören die Berechnung der Starttaxpunktwerte und der Aufbau der TrustCenter.
}

(LeiKoV) mit den Versicherern, was wiederum Grundlage für die Festlegung der Taxpunktwerte ist.»

In den vergangenen Jahren haben sich die Ansprüche an die Schweizer Ärzteschaft deutlich erhöht, und die Tarifentwicklung verlangt bei höherer Nachfrage nach immer mehr Kosteneinsparungen. Ernst Gähler, Vize-Präsident der FMH, ist überzeugt, dass die Schweizer Ärzteschaft diesen Einsparungen nur mit eigenen Daten begegnen kann. «Der umfassende Datenpool von NewIndex bietet der Schweizer Ärzteschaft die einmalige Gelegenheit, eine Leaderfunktion einzunehmen», betont Dr. Renato Laffranchi, General Manager der NewIndex AG, «denn keine andere Institution verfügt über derart detaillierte Datensätze.»

\section{OBELISC (OBjective Evaluation and Leader-} ship In Scientific Health Data Collection)

Um die steigenden Herausforderungen und auch weitere in diesem Zusammenhang stehende Aufgaben erfüllen zu können, braucht NewIndex die Mithilfe der Schweizer Ärzteschaft, um die nötigen Kompetenzen und Datengrundlagen zu schaffen, die für diese Auswertungen unverzichtbar sind. Die Schaffung des Sonderkollektivs OBELISC erlaubt NewIndex, Analysen und Auswertungen auf desaggregierten bzw. nicht verdichteten Daten durchzuführen. Unter Berücksichtigung sämtlicher Datenschutzinteressen werden alle Publikationen, die auf diesen Daten beruhen, nur mit aggregierten Daten veröffentlicht, die keinerlei Rückschlüsse auf den einzelnen Arzt oder Patienten erlauben. Es gilt also, ein neues Vertragsverhältnis zwischen Arzt und NewIndex zu schaffen, um so die Sicherheit für den Arzt zu gewährleisten: Jeder teilnehmende Arzt erlaubt NewIndex, auf seine desaggregierten Daten zuzugreifen, und NewIndex verpflichtet sich zu einer transparenten Nutzungsbindung. Der bestehende Vertrag zwischen Arzt und TrustCenter sowie die bis- 


\section{Datennutzung}

Fokus Gestern, Heute und Morgen

Einführung TARMED und KN-Phase

$>$ Verteidigung der Starttaxpunktwerte und Datenparität

LeiKoV

$>$ Kantonale TPW-Steuerung und Leistungsfaktoren

TARVISION

$>$ Revision der Tarifstruktur TARMED

nDMA

$>$ Innovatives margenunabhängiges Abgabemodell bei der ärztlichen Medikamentenabgabe

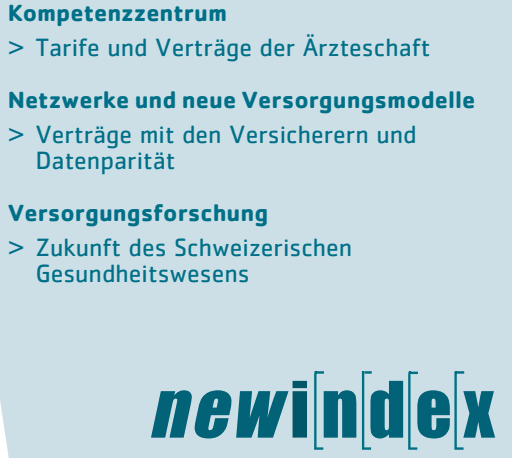

herigen Abläufe und Kontrollen (NAKO-Gremium) bleiben unangetastet und weiterhin gültig. Für die teilnehmende Ärztin oder den teilnehmenden Arzt entstehen dabei keinerlei Mehrkosten.

Die Anwesenden der Tagung vom 29.3.2012 sind sich einig: Die bereits bestehende Datenauswertung muss zukünftig optimaler genutzt werden, um Dienstleistungen im Schweizer Gesundheitswesen zu verbessern und die Interessen der Schweizer Ärzteschaft bestmöglich zu vertreten. Denn im Zentrum steht schliesslich der Nutzen für die Patienten!

\section{Desaggregierte Daten und ihre Bedeutung im schweizerischen Gesundheitswesen}

An der Tagung wurde das vorgestellte Sonderkollektiv OBELISC sehr positiv aufgenommen. Die Ausführungen von Dr. Andreas Bänziger, wissenschaftlicher Mitarbeiter an der Zürcher Hochschule für Angewandte Wissenschaften, verdeutlichen am Beispiel des «Regionalen Psychiatrischen Versorgungsbedarfs» die Wichtigkeit von nicht verdichteten Daten, die, im Gegensatz zu Daten des Tarifpools der santésuisse, eine detaillierte Analyse der Patientenanzahl auf kantonaler Ebene ermöglichen. Wichtig ist laut Bänziger die einfache Zugänglichkeit der Daten, die Freiheit der Datenauswertung und die vollständige Abdeckung der Datensätze.

Dr. Peter Wiedersheim, Co-Präsident der Konferenz der Kantonalen Ärztegesellschaften, sieht ein riesiges Potential in den erweiterten Nutzungsmöglichkeiten der erhobenen Abrechnungsdaten via die ärzteeigenen TrustCenter: umfassende Datenanalyse pro Kanton, Kontrolle bestimmter Leistungen (z.B. FSME) sowie die Beobachtung der Entwicklungen in einzelnen Fachgesellschaften oder das Abrechnungsverhalten in einzelnen Kantonen. «Wir verfügen bereits über einen ausgezeichneten Datenpool wir müssen diese Basis unbedingt erweitern und optimal nutzen, denn in Zukunft werden eigene Daten für die Ärzteschaft immer wichtiger», sagt Wiedersheim und fordert die Ärzteschaft auf, NewIndex und das Projekt OBELISC zu unterstützen.

Die Wichtigkeit ärzteeigener Daten für den Kanton und die politische Argumentation wurde von Dr. Jürg Lareida, Tarifdelegierter des Kantons Aargau, anhand der Situation im Kanton Aargau erörtert. Auf der Basis verschiedener Datenquellen konnte ein sehr vollständiges Bild für den Kanton aufgezeigt werden: die Grundversorger-Praxen sehen sich mit einer Zunahme der Kosten (in erster Linie Löhne) und sinkenden Gewinnen bei steigender Arbeitszeitbelastung konfrontiert. Dank dem Datenpool von NewIndex, so Lareida, können z. B. die Kosten nach Patientenalter gesplittet werden und erlauben so eine differenzierte Sicht. «Angesichts der zukünftigen Herausforderungen für die Schweizer Ärzteschaft wird es notwendig, neue langfristige Finanzierungslösungen für Datensammlung und Datennutzung zu diskutieren», so Anton Prantl, Direktionspräsident der Ärztekasse.

\section{Stellungnahmen von Dr. Franziska Zogga und Dr. Jürg Nadigb}

a Vorstandsmitglied Hausärzte Schweiz (Berufsverband der Haus- und Kinderärztinnen Schweiz) und Vizepräsidentin Ärzte-Gesellschaft des Kantons Zug

b Präsident Swiss Federation Of Specialities In Medicine (SFSM)

\section{Weshalb sind eigene Daten für die Schweizer Ärzteschaft wichtig?}

Zogg: Nur mit eigenen Daten können wir die Statistiken von anderen, z.B. diejenigen der santésuisse, plausibilisieren und falsche Behauptungen widerlegen. Eigene Daten helfen auch, Trends früh zu erkennen und entsprechend zu handeln.

Nadig: Dank den NewIndex-Daten konnte am Beispiel der Medizinischen Onkologen gezeigt werden, dass die von der santésuisse verwendeten Modelle zur Beurteilung der Wirtschaftlichkeit nicht in der Lage sind, die Praxisbesonderheiten abzubilden und homogene Kollektive zu bilden. Das von der Fachgesellschaft entwickelte Modell liess sich erfolgreich an den ärzteeigenen Daten validieren, und erlaubt, mit relevanten Markerpositionen homogene Kollektive zu bilden.

Welchen Stellenwert hat OBELISC für die Fachärzte resp. für Sie persönlich, und wo sehen Sie das Potential von Datensammlungen für zukünf- 
tige Herausforderungen im Schweizerischen Gesundheitswesen?

Zogg: OBELISC ist für mich ein Quantensprung: Damit sind Aussagen über den Inhalt von Sitzungen im Zusammenhang mit bestimmten Tarifpositionen oder mit der Reason for Encounter RFE möglich. Das riesige Potential in unseren Daten können wir damit erst wirklich ausschöpfen. Als Beispiel: Welche Positionen enthält ein Besuch im Durchschnitt in welcher Landesgegend? Wie unterscheiden sich Konsultationen auf dem Land von denjenigen in der Stadt? Wir werden nicht nur die Anzahl Sitzungen mit einem bestimmten RFE-Kode zählen, sondern zusätzlich auswerten können, was in diesen Sitzungen geleistet wird.

Nadig: OBELISC erlaubt eine vertiefte Analyse der Daten und somit mehr Transparenz bei den Praxisbesonderheiten. Mit den desaggregierten Daten lässt sich neu auch das Feld der Versorgungsforschung be-
Sonderkollektiv OBELISC: Machen Sie mit! Mit dem Beitritt zu OBELISC und damit der Unterzeichnung der entsprechenden Erklärung zur Freigabe Ihrer Daten ermöglichen Sie der NewIndex, die wichtigen Auswertungen im Auftrag und zum Wohle der Ärzteschaft wahrzunehmen. Falls auch Sie bei diesem zukunftsorientierten Projekt mitmachen wollen, finden Sie die Beitrittserklärung auf www.newindex.ch/d/obelisc

Welchen Nutzen sehen Sie in OBELISC und aus welchen Gründen können Sie dieses Projekt Ihren Kolleginnen und Kollegen empfehlen?

Nadig: Nachdem uns diese Daten bei den Wirtschaftlichkeitsverfahren eine Führungsrolle ermöglicht haben, sollten wir sie auch im Rahmen der Versor-

«Nur mit eigenen Daten können wir die Statistiken von anderen, z. B. diejenigen der santésuisse, plausibilisieren und falsche Behauptungen widerlegen.»

setzen. Bei zunehmendem Kostendruck wird es für die Ärzteschaft immer wichtiger, auch in diesem Bereich eigene Daten zu haben. Nur wenn wir die Qualität und die Vollkosten über eine ganze Behandlungskette kennen, können wir beginnen, die Prozesse zu steuern. gungsforschung erlangen und sie nicht den Ökonomen überlassen. Wir sind als Ärzte und Fachgesellschaften am besten in der Lage, die Daten zu analysieren. Wenn wir keine eigenen Daten haben, werden andere Gruppen ihre Daten in ihrem Interesse interpretieren. 